August 28, 2018

LBNL-40331

UCB-PTH-97/23

\title{
New Mechanism of Flavor Symmetry Breaking from Supersymmetric Strong Dynamics的
}

\author{
Christopher D. Carone ${ }^{1}$, Lawrence J. Hall ${ }^{1,2}$, and Takeo Moroi ${ }^{1}$ \\ 1 Theoretical Physics Group \\ Ernest Orlando Lawrence Berkeley National Laboratory \\ University of California, Berkeley, California 94720 \\ ${ }^{2}$ Department of Physics \\ University of California, Berkeley, California 94720
}

\begin{abstract}
We present a class of supersymmetric models in which flavor symmetries are broken dynamically, by a set of composite flavon fields. The strong dynamics that is responsible for confinement in the flavor sector also drives flavor symmetry breaking vacuum expectation values, as a consequence of a quantum-deformed moduli space. Yukawa couplings result as a power series in the ratio of the confinement to Planck scale, and the fermion mass hierarchy depends on the differing number of preons in different flavor symmetry-breaking operators. We present viable non-Abelian and Abelian flavor models that incorporate this mechanism.
\end{abstract}

\footnotetext{
${ }^{*}$ This work was supported in part by the Director, Office of Energy Research, Office of High Energy and Nuclear Physics, Division of High Energy Physics of the U.S. Department of Energy under Contract DE-AC03-76SF00098. LJH was also supported in part by the National Science Foundation under grant PHY-95-14797.
} 


\section{Disclaimer}

This document was prepared as an account of work sponsored by the United States Government. While this document is believed to contain correct information, neither the United States Government nor any agency thereof, nor The Regents of the University of California, nor any of their employees, makes any warranty, express or implied, or assumes any legal liability or responsibility for the accuracy, completeness, or usefulness of any information, apparatus, product, or process disclosed, or represents that its use would not infringe privately owned rights. Reference herein to any specific commercial products process, or service by its trade name, trademark, manufacturer, or otherwise, does not necessarily constitute or imply its endorsement, recommendation, or favoring by the United States Government or any agency thereof, or The Regents of the University of California. The views and opinions of authors expressed herein do not necessarily state or reflect those of the United States Government or any agency thereof, or The Regents of the University of California.

Lawrence Berkeley Laboratory is an equal opportunity employer. 


\section{Introduction}

Symmetry is a powerful tool for understanding the physical world, even when the symmetry in question is known to be broken. However, many candidate fundamental theories are incomplete, or flawed, because we do not know how their symmetries are broken - the origin of symmetry breaking is perhaps the greatest gap in our understanding of nature.

The spontaneous breaking of approximate light-quark flavor symmetries in QCD, leading to light pions and kaons, is the only case in nature where we know the underlying theory of symmetry breaking [1]. The origin of $\mathrm{SU}(2)_{L} \times \mathrm{U}(1)_{Y}$ electroweak symmetry breaking, leading to the $W$ and $Z$ masses, and of the $\mathrm{U}(3)^{5}$ flavor symmetry breaking, leading to the quark and lepton masses, is unknown. There are only a few candidate field theory mechanisms for such symmetry breakings. Symmetries are apparently easily broken by the vacuum expectation values of elementary scalar fields [2], but this alone is unsatisfactory, as it does not provide an understanding for the mass scale of the associated symmetry breaking. Without such information, we do not have an understanding of the basic mass scales of nature.

The only known way to generate symmetry breaking mass scales in quantum field theory is by dimensional transmutation, frequently, but not always, involving strongly interacting dynamics. Examples of such dynamical symmetry breaking are provided by QCD, and by theories of dynamical supersymmetry breaking. In supersymmetric theories, once soft scalar masses are induced from supersymmetry breaking, gauge and global symmetries may be broken by having further interactions which evolve these squared masses negative, thus dynamically generating new symmetry breaking [3]. For example, the large top Yukawa coupling has been used to drive the Higgs mass-squared negative, breaking electroweak symmetry. Much model building has centered around this two stage breaking of symmetries: first supersymmetry is broken to generate the soft squared masses, then further, non-gauge interactions give radiative corrections so that the squared masses become negative.

In view of the importance of symmetry breaking, it is striking that certain strong supersymmetric gauge interactions necessarily force a direct breaking of symmetries [4]. This does not require supersymmetry breaking, nor any other 
interactions beyond the supersymmetric gauge interactions. I' For example, in supersymmetric QCD with an equal number of flavors and colors, the strong gauge interaction forms bound state mesons and baryons, $T$ and $B$, and induces vevs for some of their scalar components. This direct forcing of symmetry breaking offers a new avenue for exploring the origins of gauge and flavor symmetry breaking. In this paper, we use this strong dynamics to construct realistic theories of flavor.

In supersymmetric extensions of the standard model, flavor symmetries are in general broken by squark and slepton mass matrices, $m^{2}$, as well as by Yukawa matrices, $h$, which generate the quark and lepton masses. In this paper we study theories where the form of both $m^{2}$ and $h$ are governed by some fundamental global flavor symmetry group, $G_{F}$, and its breaking pattern. We take the preons, $p$, and the bound states, $T$ and $B$, of some new strong gauge force to transform non-trivially under $G_{F}$. The theory contains the most general set of interactions which are gauge and $G_{F}$ invariant, both $\mathrm{F}$ and $\mathrm{D}$ terms, including non-renormalizable operators, scaled by inverse powers of the cutoff $M_{*}$, which we take to be the reduced Planck scale $M_{P l} / \sqrt{8 \pi} \simeq 2.4 \times 10^{18} \mathrm{GeV}$. In the fundamental theory, the scalar mass and Yukawa matrices can be written as field dependent polynomials, $m^{2}\left(p / M_{*}\right)$ and $h\left(p / M_{*}\right)$, where $p$ is a preon field. At the scale $\Lambda$ of the new strong force, these matrices become polynomials in the meson and baryon fields,

$$
\begin{aligned}
m^{2} & =m^{2}\left(\frac{\Lambda T}{M_{*}^{2}}, \frac{\Lambda^{N-1} B}{M_{*}^{N}}\right) \\
h & =h\left(\frac{\Lambda T}{M_{*}^{2}}, \frac{\Lambda^{N-1} B}{M_{*}^{N}}\right),
\end{aligned}
$$

where $N$ is the number of preons in a baryon. The new strong force constrains $T$ and $B$ to acquire vevs so that these fields become flavon fields, spontaneously breaking the flavor group $G_{F}$. However, there is a large vacuum degeneracy, so that $m^{2}$ and $h$ become functions on the moduli space. The main phenomenological problem is to lift this vacuum degeneracy, so that for a certain choice of $T, B$ and $G_{F}$, (1.1) and (1.2) give realistic masses.

In the next section we elaborate on the framework for symmetry breaking and solving the vacuum alignment problem. In Sections 3 and 4 we give explicit

\footnotetext{
${ }^{\dagger}$ In certain other theories, symmetry breaking can occur by the combination of supersymmetric gauge interactions and superpotential interactions. These have recently been used to study the breaking of grand unified symmetries [5].
} 


\begin{tabular}{c|c|cccc}
\hline \hline & $\mathrm{SU}(\mathrm{N})$ & $\mathrm{SU}(\mathrm{N})_{p}$ & $\mathrm{SU}(\mathrm{N})_{\bar{p}}$ & $\mathrm{U}(1)_{B}$ & $\mathrm{U}(1)_{R}$ \\
\hline$p$ & $\square$ & $\square$ & 1 & 1 & 0 \\
$\bar{p}$ & $\square$ & 1 & $\square$ & -1 & 0 \\
\hline$p \bar{p}$ & 1 & $\square$ & $\square$ & 0 & 0 \\
$p^{N}$ & 1 & 1 & 1 & $N$ & 0 \\
$\bar{p}^{N}$ & 1 & 1 & 1 & $-N$ & 0 \\
\hline \hline
\end{tabular}

Table 1: $\mathrm{SU}(\mathrm{N})$ with $N$ flavors.

realistic theories of flavor, based on non-Abelian and Abelian $G_{F}$, respectively. Our vacuum alignment mechanism results in all non-zero vevs of $T$ and $B$ being of order $\Lambda$. The flavor group is broken at a single scale - there is no hierarchy of symmetry breaking scales - so that all the small parameters of $m^{2}$ and $h$ are derived from $\Lambda / M_{*}$. For example, a term in (1.1) or (1.2) involving $n_{T}$ meson fields and $n_{B}$ baryon fields leads to a dimensionless coefficient of size $\left(\Lambda / M_{*}\right)^{2 n_{T}+N n_{B}}$. The hierarchy of quark and lepton masses arises because of the small value of $\Lambda / M_{*}$, because mesons and baryons contain different numbers of preons, and because the $G_{F}$ quantum number assignments lead to interactions with differing numbers of mesons and baryons.

\section{Framework}

In this section we outline our general approach for breaking flavor symmetries dynamically in models with composite flavon fields. We give explicit examples of viable models that incorporate this mechanism in the following section.

The sector of the theory that is responsible for confinement is a supersymmetric $\mathrm{SU}(\mathrm{N})$ gauge theory with $N$ flavors. The nonanomalous global symmetries of the theory are $G=\mathrm{SU}(\mathrm{N})_{p} \times \mathrm{SU}(\mathrm{N})_{\bar{p}} \times \mathrm{U}(1)_{B} \times \mathrm{U}(1)_{R}$, where the first $\mathrm{U}(1)$ factor is the analog of baryon number in ordinary QCD, and the second $\mathrm{U}(1)$ is an Rsymmetry. The transformation properties of the preons and their bound states under the global symmetries $G$ are shown in Table 11. Notice that there are $N^{2}$ meson fields with zero baryon number, transforming as an $(N, N)$ under the two global SU(N) groups, and a baryon-antibaryon pair that are singlets under the two $\mathrm{SU}(\mathrm{N}) \mathrm{s}$.

This confining theory has two features that are particularly relevant to model 
building. First, an $\mathrm{SU}(\mathrm{N})$ gauge theory with $N$ flavors has no dynamically generated superpotential. This follows from the fact that all the preons in Table 1 have R-charge 0 , so that it is not possible to write down an invariant combination of the fields that have R-charge 2. Secondly, the vacuum manifold of the theory is distorted by quantum mechanical effects so that the origin of field space is excluded [1]. Classically, we have the identity

$$
\operatorname{det}(p \bar{p})-p^{N} \bar{p}^{N}=0
$$

which we can rewrite in terms of canonically normalized meson and baryon fields as

$$
\operatorname{det} M-\Lambda^{N-2} B \bar{B}=0 \text {. }
$$

Quantum mechanically, this relation is modified, and becomes

$$
\operatorname{det} M-\Lambda^{N-2} B \bar{B}=\Lambda^{N}
$$

Notice that there is no symmetry which prevents the right-hand side of Eq. (2.3) from becoming nonzero. Furthermore this modified constraint is necessary if we are to properly recover the Affleck-Dine-Seiberg superpotential [6] when we decouple one flavor, beginning with the $\mathrm{SU}(\mathrm{N})$ theory with equal numbers of flavors and colors.

We learn from Eq. (2.3) that some of the meson and baryon fields acquire vevs, breaking the original global symmetry $G$. If the preons transform nontrivially under a flavor symmetry group $G_{F}$, then meson and baryon vevs may break the flavor symmetry as well. If we interpret $G$ as an accidental symmetry of the sector responsible for confinement, while $G_{F}$ is respected by all the interactions of the theory, then some of the mesons and baryons may couple to ordinary matter and serve as flavon fields. Yukawa couplings may arise via Planck-suppressed operators, as described in Section 1, so that the small parameter that characterizes flavor symmetry breaking is the ratio of the confinement scale $\Lambda$ to the reduced Planck mass $M_{*}$.

The ambiguity that must now be resolved is the precise set of composites that actually acquire vevs. For example, Eq. (2.3) is satisfied by a point in field space where the baryons $B$ and $\bar{B}$ acquire confinement-scale vevs, while the mesons remain at the origin. This vacuum would not be particularly useful if we were to construct a model in which only the mesons coupled to ordinary matter. In 
a viable model, we must sufficiently reduce this vacuum degeneracy so that the flavor symmetry breaking fields which couple to ordinary matter are forced to get vevs. The models that we present in the next section achieve this in two steps:

First, we introduce additional fields $X_{j}(j=0,1,2, \ldots)$, that couple to the preons via nonrenormalizable superpotential interactions. Since the preon fields have $\mathrm{U}(1)_{R}$ charge 0 , we will take the fields $X$ to have $R$-charge 2 . We impose the $\mathrm{U}(1)_{R}$ symmetry so that all of the preonic operators involve one of the $X$ fields. We will assume that $X_{0}$ is a singlet under the non- $R$ symmetries shown in Table 1, while the remaining $X_{j}$ transform nontrivially under $G_{F}$. The $X$ fields will be responsible for restricting the moduli space such that the desired set of mesons and baryons develop vacuum expectation values when the scalar potential is minimized.

The F-flatness conditions for the $X$ fields significantly reduce the original supersymmetric vacuum degeneracy. Consider the superpotential interactions for the field $X_{0}$. In the models of interest, these will be of the form

$$
W_{0}=\left(\frac{1}{M_{*}}\right)^{2 N-2}\left(\frac{\Lambda}{M_{*}}\right)^{N}\left[\sum_{j} G_{j}+\Lambda^{N-2} B \bar{B}\right] X_{0}
$$

where the $G_{i}$ represent all possible flavor-group invariant combinations of the meson fields involving $2 N$ preons. In the models we will consider, these interactions will be the ones of lowest order in $1 / M_{*}$ that are allowed by the flavor symmetry. Other interactions, such as direct $G_{F}$-invariant couplings between the baryons and mesons, will arise at higher order, and will be suppressed. Note that we have omitted a Planck-scale linear term for $X_{0}$, which can be forbidden by imposing an anomalous discrete symmetry, as we will see explicitly in the next section. Notice that the F-flatness condition for $X_{0}$ together with the quantummodified constraint (2.3) yield two restrictions on the set of invariants $G_{i}, B \bar{B}$. Thus, we have succeeded in reducing the vacuum degeneracy by one degree of freedom. The $X_{0}$ field orients the vacuum so that at least some of the mesons have non-vanishing vevs.

Now we introduce additional fields $X_{j}$, that transform nontrivially under the

\footnotetext{
${ }^{\ddagger}$ We assume that this symmetry is spontaneously broken in the hidden sector, so that we generate gaugino masses, trilinear scalar interactions, etc.
} 
flavor group $G_{F}$. These lead to additional superpotential couplings of the form

$$
W_{j}=X_{j} \sum_{i} G_{i}^{\prime}
$$

where the $G_{i}^{\prime}$ represent all possible baryon and meson interactions with the appropriate quantum numbers to couple to $X_{j}$. We have absorbed powers of $\Lambda$ and $M_{*}$ into the definition of the $G_{i}^{\prime}$ for notational convenience. In a successful model, we introduce enough nontrivial constraints in this way such that the flavor invariant combinations of the mesons and baryons shown in brackets in Eq. (2.4) acquire vevs individually,

$$
\begin{gathered}
B \bar{B} \sim \Lambda^{2} \\
G_{1} \sim \Lambda^{N} \\
G_{2} \sim \Lambda^{N} \\
\text { etc. }
\end{gathered}
$$

while all the $X$ field vevs vanish. This result should remain valid provided that the Kähler metric is positive definite in the region of field space where we have located the minimum. Since the Kähler potential is not calculable for field amplitudes of the same order as the confinement scale, we take this positivity requirement as a mild assumption. Note also that if too many F-flatness constraints are added, it is possible that the resulting superpotential may break supersymmetry. This would lead to direct, flavor-dependent couplings of fields with large F components to ordinary matter, which would not be desirable. In all the models we consider, supersymmetry will remain unbroken after the effects of the $X$ fields are taken into account.

Once we have arranged for each gauge invariant combination of the mesons and baryons to acquire vevs, we must lift the remaining vacuum degeneracy. Notice that given any point in the moduli space defined by Eq. (2.6), we can reach another point by transforming the fields under the complexification of the flavor group. If we include positive soft supersymmetry-breaking squared masses for the the composite flavon fields, the complexified symmetry will be broken, and this last flat direction will be lifted. (We justify this procedure below.) To make this point concrete, imagine we have a theory with three flavons, $\phi_{0}, \phi_{+}$and $\phi_{-}$, where the subscript indicates the charge under some $U(1)$ symmetry. Now assume that the moduli space is constrained such that $\phi_{0}^{3} \sim \Lambda^{3}$ and $\phi_{0} \phi_{+} \phi_{-} \sim \Lambda^{3}$. 
The remaining flat direction corresponds to a rescaling of $\phi_{+}$and $\phi_{-}$, which is generated by the complexification of the U(1) symmetry. The complexified symmetry is explicitly broken by the soft mass terms $V_{\text {soft }}=m_{\text {soft }}^{2}\left(\left|\phi_{0}\right|^{2}+\left|\phi_{+}\right|^{2}+\right.$ $\left|\phi_{-}\right|^{2}$ ), and minimization of the full potential then yields $\phi_{+} \sim \phi_{-} \sim \phi_{0} \sim \Lambda$, as desired.

This last step may be questioned since the form of the soft supersymmetry breaking interactions in the confining theory are not determined by any symmetry argument. However, we may justify our qualitative result by considering the behavior of the theory in the limit of large field amplitudes. Our constraints on the gauge-invariant products of the fields $G_{i} \sim \Lambda^{N}$ imply that varying any moduli field away from $\Lambda$ forces some field to acquire a vev greater than $\Lambda$. In the limit of large field amplitudes, this corresponds to at least some of the preons $p$ acquiring large expectation values as well, $p>\Lambda$. In the same limit we expect there will be soft supersymmetry breaking squared masses for the preon fields, so $V_{\text {soft }} \sim m_{\text {soft }}^{2}|p|^{2}$. Again assuming positive $m_{\text {soft }}^{2}$, the potential grows as we take any $p$ larger than $\Lambda$, and we conclude that our previous result is energetically favored. This conclusion is consistent with the assumption made in Ref. [7] that minimization of a potential that includes soft supersymmetry breaking masses for the composite fields should lead to correct qualitative results, even when field amplitudes are of the same order as the confinement scale. Therefore, for the purpose of calculation, we will assume soft masses for the composite fields, but the reader should keep in mind that the results are supported by this more general argument.

Finally, we will make the simplifying assumption that trilinear scalar interactions (A-terms) can be neglected in the potential. Any minimum of the potential that we find in the absence of A-terms will remain at least a local minimum for small but nonvanishing A parameters. This will be sufficient for our purposes. We will not attempt to find the explicit conditions implied by vacuum stability on the possible trilinear scalar interactions in the flavor sector when the $A$ parameters are large.

After taking into account both the F-flatness conditions for the $X$ fields, and the effect of soft supersymmetry-breaking scalar masses, it is often the case that the desired composite fields will each be forced to acquire a vev of order $\Lambda$. We will now present two complete models that successfully incorporate the flavor symmetry breaking mechanism described in this section. 


\section{Non-Abelian Model}

The models we present in this and the next section are based on $\mathrm{SU}(3)$ supersymmetric QCD with three flavors. The global symmetries of the strong interaction are

$$
G=\mathrm{SU}(3)_{p} \times \mathrm{SU}(3)_{\bar{p}} \times \mathrm{U}(1)_{B} \times \mathrm{U}(1)_{R}
$$

and

$$
G_{A}=\mathrm{U}(1)_{A}
$$

where $G_{A}$ is the anomalous $\mathrm{U}(1)$ symmetry corresponding to axial phase rotations on $p$ and $\bar{p}$. In each of the models we present, the action of the flavor group $G_{F}$ on the preon fields will be isomorphic to a subgroup of $G \times G_{A}$. However, one should keep in mind that the ordinary fermions will transform under $G_{F}$ even though they do not transform under the global symmetries of the confining flavor sector.

The flavor group of the first model is

$$
G_{F}=\mathrm{SU}(2)_{F} \times \mathrm{U}(1)_{F} \times \mathrm{U}(1)_{F^{\prime}} \times Z_{2}
$$

The transformation properties of the MSSM superfields (as well as those of the composite states discussed later) are shown in Table \&. The lighter two generations of the matter fields $\left(Q^{i}, U^{i}\right.$, and $D^{i}$, with $\left.i=1,2\right)$ transform as doublets under $\mathrm{SU}(2)_{F}$, while the third generation fields $\left(Q^{3}, U^{3}\right.$, and $\left.D^{3}\right)$ are singlets. The $\mathbf{2}+\mathbf{1}$ representation structure provides a natural degeneracy between squark masses of the first and second generations in the flavor symmetric limit [8]. This leads to a suppression of flavor changing neutral current effects when the flavor symmetries are broken. The remaining group factors, $\mathrm{U}(1)_{F} \times \mathrm{U}(1)_{F^{\prime}}$, are used to obtain realistic Yukawa textures. The fields $Q^{i}, U^{i}, D^{i}$, and $D^{3}$ transform nontrivially under the two flavor $\mathrm{U}(1)$ factors, while $Q^{3}, U^{3}$ and the ordinary Higgs fields are $G_{F}$ invariant. The top quark Yukawa coupling is invariant under the flavor symmetry, and hence can be of order one, while the other Yukawa elements will be suppressed by the ratios of flavon vevs to $M_{*}$.

If we consider the preonic sector alone, the flavor symmetry can be identified with a subgroup of $G \times G_{A}$. We first decompose each $\mathrm{SU}(3)$ factor into its $\mathrm{SU}(2) \times \mathrm{U}(1)$ subgroup:

$$
\mathrm{SU}(3)_{p} \times \mathrm{SU}(3)_{\bar{p}} \times \mathrm{U}(1)_{B} \rightarrow[\mathrm{SU}(2) \times \mathrm{U}(1)]_{p} \times[\mathrm{SU}(2) \times \mathrm{U}(1)]_{\bar{p}} \times \mathrm{U}(1)_{B}
$$




\section{MSSM Fields}

\begin{tabular}{l|ll}
\hline \hline & $\mathrm{G}_{\mathrm{SM}}$ & $\mathrm{G}_{\mathrm{F}}$ \\
\hline$Q^{i}$ & $\left(\square, \square, \frac{1}{6}\right)$ & $(\bar{\square},-1,2,-)$ \\
$Q^{3}$ & $\left(\square, \square, \frac{1}{6}\right)$ & $(\mathbf{1}, 0,0,+)$ \\
$U^{i}$ & $\left(\bar{\square}, \mathbf{1},-\frac{2}{3}\right)$ & $(\bar{\square},-1,2,-)$ \\
$U^{3}$ & $\left(\bar{\square} \mathbf{1},-\frac{2}{3}\right)$ & $(\mathbf{1}, 0,0,+)$ \\
$D^{i}$ & $\left(\bar{\square}, \mathbf{1}, \frac{1}{3}\right)$ & $(\bar{\square},-1,2,-)$ \\
$D^{3}$ & $\left(\bar{\square}, \mathbf{1}, \frac{1}{3}\right)$ & $(\mathbf{1}, 2,2,-)$ \\
\hline
\end{tabular}

\section{Composite Fields}

\begin{tabular}{c|ll}
\hline \hline & $\mathrm{G}_{\mathrm{SM}}$ & $\mathrm{G}_{\mathrm{F}}$ \\
\hline$\phi_{i}$ & $(\mathbf{1}, \mathbf{1}, 0)$ & $(\square, 1,-2,-)$ \\
$\tilde{\phi}_{i}$ & $(\mathbf{1}, \mathbf{1}, 0)$ & $(\square,-2,1,-)$ \\
$A$ & $(\mathbf{1}, \mathbf{1}, 0)$ & $(\mathbf{1}, 1,1,-)$ \\
$S_{i j}$ & $(\mathbf{1}, \mathbf{1}, 0)$ & $(\square, 1,1,-)$ \\
$\sigma$ & $(\mathbf{1}, \mathbf{1}, 0)$ & $(\mathbf{1},-2,-2,-)$ \\
$B$ & $(\mathbf{1}, \mathbf{1}, 0)$ & $(\mathbf{1}, 1,-2,-)$ \\
$\bar{B}$ & $(\mathbf{1}, \mathbf{1}, 0)$ & $(\mathbf{1},-1,2,+)$ \\
\hline \hline
\end{tabular}

Table 2: The transformation properties of the quarks and the composite states under the standard model gauge group $G_{\mathrm{SM}}=\mathrm{SU}(3)_{C} \times \mathrm{SU}(2)_{L} \times \mathrm{U}(1)_{Y}$ and the flavor symmetry $G_{F}=\mathrm{SU}(2)_{F} \times \mathrm{U}(1)_{F} \times \mathrm{U}(1)_{F^{\prime}} \times Z_{2}$. Here, $i=1,2$ is the $\mathrm{SU}(2)_{F}$ index. Note that the Higgs fields $H_{1}$ and $H_{2}$ are invariant under $\mathrm{G}_{\mathrm{F}}$. 
The flavor $\mathrm{SU}(2)$ is simply the diagonal subgroup of $\mathrm{SU}(2)_{p} \times \mathrm{SU}(2)_{\bar{p}}$. The two flavor $\mathrm{U}(1)$ factors are different linear combinations of $\mathrm{U}(1)_{B}, \mathrm{U}(1)_{p}$ and $\mathrm{U}(1)_{\bar{p}}$. The charges under the flavor U(1)s are defined by

$$
Q_{\mathrm{F}}=2 \sqrt{3} Q_{p}+\frac{1}{3} Q_{\mathrm{B}} \quad \text { and } \quad Q_{\mathrm{F}^{\prime}}=2 \sqrt{3} Q_{\bar{p}}-\frac{2}{3} Q_{\mathrm{B}},
$$

where $Q_{p}$ and $Q_{\bar{p}}$ are the eigenvalues of the $T^{8}$ generators of $\mathrm{SU}(3)_{p}$ and $\mathrm{SU}(3)_{\bar{p}}$, respectively.

The quantum numbers of the preons $p$ and $\bar{p}$ under $\mathrm{SU}(3) \times G_{F}$ are given by

$$
\begin{aligned}
p^{i} & \sim\left(\square, \square, \frac{4}{3},-\frac{2}{3},-\right) \\
p & \sim\left(\square, \mathbf{1},-\frac{5}{3},-\frac{2}{3},-\right) \\
\bar{p}^{i} & \sim\left(\bar{\square}, \square,-\frac{1}{3}, \frac{5}{3},+\right) \\
\bar{p} & \sim\left(\bar{\square}, \mathbf{1},-\frac{1}{3}, \frac{4}{3},+\right) .
\end{aligned}
$$

Notice that the $Z_{2}$ factor is a symmetry under which all the preons are odd and all anti-preons are even; this is a discrete subgroup of $\mathrm{U}(1)_{A} \times \mathrm{U}(1)_{B}$. Once the $\mathrm{SU}(3)$ gauge group becomes strong at the scale $\Lambda$, the preons form composite states:

$$
\begin{aligned}
S_{i j} & \sim \Lambda^{-1}\left(p_{i} \bar{p}_{j}+p_{j} \bar{p}_{i}\right), \\
A & \sim \Lambda^{-1} \epsilon^{i j}\left(p_{i} \bar{p}_{j}\right), \\
\phi_{i} & \sim \Lambda^{-1}\left(p_{i} \bar{p}\right), \\
\tilde{\phi}_{i} & \sim \Lambda^{-1}\left(p \bar{p}_{i}\right), \\
\sigma & \sim \Lambda^{-1}(p \bar{p}), \\
B & \sim \Lambda^{-2} \epsilon^{i j}\left(p_{i} p_{j} p\right), \\
\bar{B} & \sim \Lambda^{-2} \epsilon^{i j}\left(\bar{p}_{i} \bar{p}_{j} \bar{p}\right),
\end{aligned}
$$

where $i$ and $j$ are $\mathrm{SU}(2)_{F}$ flavor indices. The composite fields have been given canonical mass dimension by including appropriate powers of $\Lambda^{-1}$. The transformation properties of the composite states under the flavor symmetry are also summarized in Table 2.

Given these quantum number assignments, and our assumption that Planckscale physics induces all operators that are consistent with the symmetries, some 
of the composite states above can serve as flavon fields. The $G_{F}$-allowed couplings that can contribute to the Yukawa matrices are summarized as follows:

$$
\begin{gathered}
h_{u} \sim\left(\begin{array}{cc|c}
0 & B^{2} & 0 \\
-B^{2} & \phi_{2} \phi_{2} & \phi_{2} \\
\hline 0 & \phi_{2} & 1
\end{array}\right) \\
h_{d} \sim\left(\begin{array}{cc|c}
0 & B^{2} & 0 \\
-B^{2} & \phi_{2} \phi_{2} & \phi_{2} \sigma \\
\hline 0 & \phi_{2} & \sigma
\end{array}\right) .
\end{gathered}
$$

We have not shown couplings to $\phi_{1}$, since we will always work in a basis where the $\phi_{1}$ vev vanishes. We have also temporarily suppressed the factors of $\Lambda$ and $M_{*}$ in each entry, which depend on the dimensionality of the original preonic interaction. Note that if a composite field above acquires a vev of order $\Lambda$, then the size of the corresponding Yukawa entry will be $\left(\Lambda / M_{*}\right)^{n}$, where $n$ is the total number of preons involved in the preonic higher-dimension operator. To obtain a realistic theory we need only lift the vacuum degeneracy such that $B, \phi$ and $\sigma$ are all forced to acquire vevs of order $\Lambda$. There may be many ways to accomplish this; below we provide an explicit example.

Since the confining sector of our model is of the type described in Section 2, the moduli space of the composite states is restricted by a quantum-modified constraint [4]. The important point is that the origin of field space is excluded, so that the flavor symmetries are guaranteed to break. The constraint is realized by a dynamically generated Lagrange multiplier term in the superpotential

$$
\begin{aligned}
W_{\mathrm{dyn}}= & \eta\left[C _ { M ^ { 3 } } \left(\epsilon^{i j} A \phi_{i} \tilde{\phi}_{j}+A^{2} \sigma+\epsilon^{i j} \epsilon^{k l} S_{i k} \phi_{j} \tilde{\phi}_{l}\right.\right. \\
& \left.\left.+\epsilon^{i j} \epsilon^{k l} S_{i k} S_{j l} \sigma\right)+C_{B \bar{B}} \Lambda B \bar{B}-\Lambda^{3}\right],
\end{aligned}
$$

where $\eta$ is the Lagrange multiplier field, and $C$ 's are $\mathcal{O}(1)$ coefficients that arise from the dynamics of confinement.

As we described in Section 2, the constraint equation alone leaves us with a rather large vacuum degeneracy, and the possibility that we will not obtain a viable pattern of flavor symmetry breaking. We will remove most of these flat directions by introducing several $X$ fields, to place additional constraints on the composite states. Perhaps the simplest set of $X$ fields is given by

$$
X_{0} \sim(\mathbf{1}, 0,0,-)
$$




$$
\begin{aligned}
& X_{1} \sim(\mathbf{1}, 1,1,+) \\
& X_{2} \sim(\square,-1,-1,-),
\end{aligned}
$$

where we have shown the transformation properties under $G_{F}$ in parentheses. We can now write down the following interaction terms for the preons:

$$
\begin{aligned}
W_{0}= & \frac{1}{M_{*}^{4}} X_{0}\left[\left(\epsilon^{i j} p_{i} \bar{p}_{j}\right) \epsilon^{k l}\left(p_{k} \bar{p}\right)\left(p \bar{p}_{l}\right)+\left(\epsilon^{i j} p_{i} \bar{p}_{j}\right)^{2}(p \bar{p})+\epsilon^{i j} \epsilon^{k l}\left(p_{i} \bar{p}_{k}+p_{k} \bar{p}_{i}\right)\left(p_{j} \bar{p}\right)\left(p \bar{p}_{l}\right)\right. \\
& \left.+\epsilon^{i k} \epsilon^{j l}\left(p_{i} \bar{p}_{j}+p_{j} \bar{p}_{i}\right)\left(p_{k} \bar{p}_{l}+p_{l} \bar{p}_{k}\right)(p \bar{p})+\left(\epsilon^{i j} p_{i} p_{j} p\right)\left(\epsilon^{k l} \bar{p}_{k} \bar{p}_{l} \bar{p}\right)\right] \\
W_{1}= & \frac{1}{M_{*}^{2}} X_{1}\left[\left(\epsilon^{i j} p_{i} \bar{p}_{j}\right)(p \bar{p})+\epsilon^{i j}\left(p_{i} \bar{p}\right)\left(p \bar{p}_{j}\right)\right] \\
W_{2}= & \epsilon^{i k} \epsilon^{j l} X_{2, i j}\left(p_{k} \bar{p}_{l}+p_{l} \bar{p}_{k}\right),
\end{aligned}
$$

where we have omitted unknown $\mathcal{O}(1)$ coefficients. Notice that there is no linear term in $X_{0}$ due to the discrete $Z_{2}$ symmetry. Without this symmetry, the interaction $M_{*}^{2} X_{0}$ would also be allowed, and the argument presented below would break down. Note that the $Z_{2}$ symmetry has no significant effect on the mass matrix textures that we obtain in either of our models.

After confinement, F-flatness conditions for the Lagrange multiplier field $\eta$ and the fields $X_{j}$ give us the following four equations of motion for composite states:

$$
\begin{aligned}
& C_{M^{3}} \epsilon^{i j} A \phi_{i} \tilde{\phi}_{j}+C_{M^{3}} A^{2} \sigma+C_{B \bar{B}} \Lambda B \bar{B}-\Lambda^{3}=0, \\
& C_{A \phi \tilde{\phi}}^{\prime} \epsilon^{i j} A \phi_{i} \tilde{\phi}_{j}+C_{A^{2} \sigma}^{\prime} A^{2} \sigma+C_{B \bar{B}}^{\prime} \Lambda B \bar{B}-\Lambda^{3}=0, \\
& C_{\phi \tilde{\phi}}^{\prime \prime} \epsilon^{i j} \phi_{i} \tilde{\phi}_{j}+C_{A \sigma}^{\prime \prime} A \sigma=0 \\
& S_{i j}=0 .
\end{aligned}
$$

Here the $C^{\prime}$ and $C^{\prime \prime}$ are also $\mathcal{O}(1)$ coefficients ${ }^{\text {s }}$. Note that we have dropped the terms which depend on $S_{i j}$ in Eqs.(3.23) - (3.25) by using eq.(3.26). We can easily solve eqs.(3.23) - (3.25), and we obtain

$$
\begin{aligned}
& A^{2} \sigma \sim \Lambda^{3}, \\
& \epsilon^{i j} A \phi_{i} \tilde{\phi}_{j} \sim \Lambda^{3}, \\
& B \bar{B} \sim \Lambda^{2},
\end{aligned}
$$

\footnotetext{
$\S$ In writing down the low energy description of the operator $X_{0}[p p p \bar{p} \bar{p} \bar{p}] / M_{*}^{4}$, we have included a linear term $X_{0} \Lambda^{6} / M_{*}^{4}$. This term can be justified by treating $\Lambda$ as a spurion under the anomalous axial U(1) symmetry of the dynamical sector, and including the most general set of invariant interactions. However, nothing in our analysis changes if such a term is absent.
} 
neglecting the possibility of accidental cancellations.

At this stage, the remaining flat directions correspond to rescaling of the composite fields, since only their products are constrained by Eqs. (3.27) - (3.29). One might suspect that these flat directions can be lifted by including yet higher order corrections to the superpotential. However, this can never be the case because the remaining flat directions are protected by a symmetry that is respected by all F-term contributions to the potential in the supersymmetric limit. Since our model has an $\mathrm{SU}(2)_{F} \times \mathrm{U}(1)_{F} \times \mathrm{U}(1)_{F^{\prime}}$ global symmetry, and the superpotential is holomorphic in the fields, the actual symmetry of the superpotential before supersymmetry breaking is the complexification of the flavor group. Since $\mathrm{SU}(2) \times \mathrm{U}(1) \times \mathrm{U}(1)$ has 5 generators, this symmetry corresponds to 5 complex degrees of freedom in the moduli space. We began with 9 meson and 2 baryon fields, and imposed $6 \mathrm{~F}$-flatness conditions (for the fields $\eta, X_{0}, X_{1}$, and the three components of $X_{2}$ ) leaving 5 complex degrees of freedom. Thus, we have lifted all the flat directions that are not protected by the complexified symmetry.

To lift the flat directions defined by Eqs. (3.27) - (3.29), we include the soft supersymmetry-breaking scalar masses for the composite states,

$$
V_{\text {soft }}=m_{\text {soft }}^{2}\left(|A|^{2}+|S|^{2}+|\phi|^{2}+|\tilde{\phi}|^{2}+|\sigma|^{2}+|B|^{2}+|\bar{B}|^{2}\right)
$$

with $m_{\text {soft }}^{2}>0$. We now minimize the potential above subject to the constraints (3.27) - 3.29). The qualitative result is easy to understand. We first use the $\mathrm{SU}(2)_{\mathrm{F}}$ symmetry to work in the basis where $\phi_{1}=0$. In this basis, $\tilde{\phi}_{2}$ appears in Eq. (3.30), but not in any of the constraints, and is therefore driven to zero. The non-vanishing elements $\left(\phi_{2}, \tilde{\phi}_{1}, A, \sigma, B\right.$, and $\left.\bar{B}\right)$ must all be of $\mathcal{O}(\Lambda)$, so that the constraints (3.27) - (3.29) are satisfied and $V_{\text {soft }} \sim m_{\text {soft }}^{2} \Lambda^{2}$. If any of the fields were to have a vev smaller than $\Lambda$, the constraint equations assure that another composite have a vev larger than $\Lambda$, and we would obtain $V_{\text {soft }}>m_{\text {soft }}^{2} \Lambda^{2}$. Therefore, up to an $\mathrm{SU}(2)_{\mathrm{F}}$ rotation, the minimum is at

$$
\phi \sim\left(\begin{array}{c}
0 \\
\Lambda
\end{array}\right), \quad \tilde{\phi} \sim\left(\begin{array}{c}
\Lambda \\
0
\end{array}\right), \quad A \sim \sigma \sim B \sim \bar{B} \sim \Lambda, \quad S_{i j}=0 .
$$

This result can be verified by explicit minimization of the potential, taking into account all the order one parameters. However, the estimate in Eq. (3.31) will be sufficient for our purposes.

ॠHere, we do not assume universal soft supersymmetry-breaking masses. The $m_{\text {soft }}^{2}$ in Eq. (3.30) is understood to be different for each of the terms shown. 
The vevs in Eq. (3.31) are exactly what we require to obtain viable textures from Eqs. (3.16) and (3.17). If we fix the ratio

$$
\lambda \equiv \frac{\Lambda}{M_{*}}<1
$$

we obtain

$$
h_{u} \sim\left(\begin{array}{ccc}
0 & \lambda^{6} & 0 \\
\lambda^{6} & \lambda^{4} & \lambda^{2} \\
0 & \lambda^{2} & 1
\end{array}\right), \quad h_{d} \sim\left(\begin{array}{ccc}
0 & \lambda^{6} & 0 \\
\lambda^{6} & \lambda^{4} & \lambda^{4} \\
0 & \lambda^{2} & \lambda^{2}
\end{array}\right)
$$

All the elements in the Yukawa matrices are predicted in terms of one small parameter $\lambda$, up to unknown coefficients of order one. As we will see below, Eq. (3.33) results in a realistic pattern of the quark masses and mixing angles, if $\lambda \sim 0.2-0.3$, and the unknown $\mathcal{O}(1)$ coefficients are chosen appropriately.

We will now consider the pattern of quark masses and mixing angles more carefully, beginning with the up sector. The largest element in the up quark Yukawa matrix is the $(3,3)$ entry, which is of order 1 , while the other elements are suppressed by powers of $\lambda$. Thus, $h_{u}$ has an eigenvalue close to one which we can identify with the top quark Yukawa coupling. Next, we consider the 2-3 block, since the remaining elements are much more suppressed. The determinant of this block is of $\mathcal{O}\left(\lambda^{4}\right)$ indicating there is an eigenvalue of the same order, which we identify as the charm quark Yukawa coupling. The rotation angle involved in the diagonalization of this block is $\mathcal{O}\left(\lambda^{2}\right)$, and hence $V_{c b} \sim \lambda^{2}$, if the up sector gives the dominant contribution. Finally, we see that $\operatorname{det} h_{u} \sim \mathcal{O}\left(\lambda^{12}\right)$, which implies that the smallest eigenvalue is $\mathcal{O}\left(\lambda^{8}\right)$. We identify this with the up quark Yukawa coupling. Thus, in our model we find

$$
m_{u}: m_{c}: m_{t} \sim \lambda^{8}: \lambda^{4}: 1 \quad \text { and } \quad V_{c b} \sim \lambda^{2} .
$$

As far as the mass eigenvalues and $V_{c b}$ are concerned, the result for $h_{u}$ in our model works fairly well. The only problem is that the mixing between first and second generations, i.e. the Cabibbo angle, is $\mathcal{O}\left(\lambda^{2}\right)$, which is too small if $\lambda \sim 0.2-0.3$. ( $V_{u b}$ on the other hand would be $\mathcal{O}\left(\lambda^{4}\right)$, which is acceptable.) Thus, the Cabibbo angle should have its origin in the down sector. We will come back to this point later.

We may now analyze $h_{d}$ in the same way. From the 2-3 block, we obtain the two larger eigenvalues of $h_{d}$, which are $\mathcal{O}\left(\lambda^{2}\right)$ and $\mathcal{O}\left(\lambda^{4}\right)$. We identify these as the 
Yukawa coupling of the bottom and strange quarks, respectively. Notice that, with the choice of $\tan \beta \sim 2$, we obtain the correct value of the ratio of $m_{b} / m_{t}$. The 2-3 mixing angle is again $\mathcal{O}\left(\lambda^{2}\right)$, and is consistent with the value of $V_{c b}$ for $\lambda \sim 0.2-0.3$. Finally, we must evaluate the down quark Yukawa coupling as well as the 1-2 mixing. Our results in Eq. (3.33) imply naively that the down quark Yukawa coupling is of $\mathcal{O}\left(\lambda^{8}\right)$ and the the 1-2 mixing angle of $\mathcal{O}\left(\lambda^{2}\right)$, both of which are too small to be consistent with observation. To fix this problem, we must take into account the possible fluctuations of the unknown order one coefficients. If we allow the couplings giving the $(1,2)$ and $(2,1)$ elements of Eq. (3.17) to be enhanced by a factor of $1 / \sqrt{\lambda} \sim 2$, and the $(2,2)$ and $(3,2)$ elements to be suppressed by the same amount, we will obtain a Cabibbo angle of $\mathcal{O}(\lambda)$, and a down quark coupling $\left(\lambda^{6} \sqrt{\lambda}\right)$. Note that the predicted ratio $m_{d} / m_{b} \sim \lambda^{4} \sqrt{\lambda}$ is consistent with recent lattice estimates of the down quark mass [9].

With this choice for the $\mathcal{O}(1)$ coefficients, the Yukawa matrix elements are given more accurately by

$$
h_{u} \sim\left(\begin{array}{ccc}
0 & \lambda^{6} & 0 \\
\lambda^{6} & \lambda^{4} & \lambda^{2} \\
0 & \lambda^{2} & 1
\end{array}\right), \quad h_{d} \sim\left(\begin{array}{ccc}
0 & \lambda^{11 / 2} & 0 \\
\lambda^{11 / 2} & \lambda^{9 / 2} & \lambda^{4} \\
0 & \lambda^{5 / 2} & \lambda^{2}
\end{array}\right)
$$

We will diagonalize the results shown in Eq. (3.35) when we need to evaluate a squark mass matrix in the quark mass eigenstate basis.

Finally, we present the textures for the squark mass matrices. The soft supersymmetry-breaking masses originate from D-terms interactions, which are not required to be holomorphic functions of the flavon fields. For example, the leading contributions to the left-handed squark masses are given by the operators

$$
\begin{aligned}
V_{\tilde{Q} \tilde{Q}^{*}} \sim & \tilde{m}^{2}\left[c_{0}\left|\tilde{Q}^{1}\right|^{2}+c_{0}\left|\tilde{Q}^{2}\right|^{2}+c_{3}\left|\tilde{Q}^{3}\right|^{2}\right. \\
& +\frac{\Lambda^{2}}{M_{*}^{4}}\left(\phi_{i} \tilde{Q}^{i}\right)\left(\phi_{j} \tilde{Q}^{j}\right)^{*}+\frac{\Lambda^{2}}{M_{*}^{4}}\left(\tilde{\phi}_{i} \tilde{Q}^{i}\right)\left(\tilde{\phi}_{j} \tilde{Q}^{j}\right)^{*} \\
& +\frac{\Lambda^{2}}{M_{*}^{4}}\left(\epsilon_{i j} \phi^{* i} \tilde{Q}^{j}\right)\left(\epsilon_{k l} \phi^{* k} \tilde{Q}^{l}\right)^{*}+\frac{\Lambda^{2}}{M_{*}^{4}}\left(\epsilon_{i j} \tilde{\phi}^{* i} \tilde{Q}^{j}\right)\left(\epsilon_{k l} \tilde{\phi}^{* k} \tilde{Q}^{l}\right)^{*} \\
& +\frac{\Lambda^{6}}{M_{*}^{10}}\left\{\left(\phi_{i} \tilde{Q}^{i}\right)\left(\epsilon_{j k} \phi^{* j} \tilde{Q}^{k}\right)^{*} B^{* 2}+h . c .\right\} \\
& +\frac{\Lambda^{5}}{M_{*}^{8}}\left\{\left(\epsilon_{i j} \phi^{* j} \tilde{Q}^{k}\right) \tilde{Q}^{3 *} B \bar{B}^{*}+h . c .\right\}
\end{aligned}
$$




$$
\left.+\frac{\Lambda^{2}}{M_{*}^{4}}\left\{\left(\tilde{\phi}_{i} \tilde{Q}^{i}\right) \tilde{Q}^{3 *}+\text { h.c. }\right\}\right],
$$

where $\tilde{m}$ is the typical scale of the squark masses. We have only shown order one coefficients explicitly in the flavor-invariant terms $\left(c_{0}\right.$ and $\left.c_{3}\right)$ to remind the reader that the first two generation scalars are degenerate in the flavor symmetric limit, while the third generation scalar is unconstrained. After flavor symmetry breaking, the operators above lead to the texture

$$
\left(\tilde{M}_{q}^{2}\right)_{L L}^{0} \sim \tilde{m}^{2}\left(\begin{array}{ccc}
c_{0}+\lambda^{4} & \lambda^{10} & \lambda^{8} \\
\lambda^{10} & c_{0}+\lambda^{4} & \lambda^{2} \\
\lambda^{8} & \lambda^{2} & c_{3}
\end{array}\right)
$$

where the powers of $\lambda$ indicate the correction to the flavor invariant result with $\mathcal{O}(1)$ coefficients suppressed. It is straightforward to repeat this analysis for the right-handed squarks, and we obtain

$$
\left(\tilde{M}_{u}^{2}\right)_{R R}^{0} \sim \tilde{m}^{2}\left(\begin{array}{ccc}
c_{0}^{\prime}+\lambda^{4} & \lambda^{10} & \lambda^{8} \\
\lambda^{10} & c_{0}^{\prime}+\lambda^{4} & \lambda^{2} \\
\lambda^{8} & \lambda^{2} & c_{3}^{\prime}
\end{array}\right)
$$

and

$$
\left(\tilde{M}_{d}^{2}\right)_{R R}^{0} \sim \tilde{m}^{2}\left(\begin{array}{ccc}
c_{0}^{\prime \prime}+\lambda^{4} & \lambda^{10} & \lambda^{10} \\
\lambda^{10} & c_{0}^{\prime \prime}+\lambda^{4} & \lambda^{4} \\
\lambda^{10} & \lambda^{4} & c_{3}^{\prime \prime}
\end{array}\right) .
$$

We may now consider the bounds from flavor changing neutral current processes. We define the parameters

$$
\begin{aligned}
& \left(\delta_{i j}^{q}\right)_{X X} \equiv\left|\left(\tilde{M}_{q}^{2}\right)_{X X, i j}\right| / \tilde{m}^{2} \quad(X=L, R) \\
& \bar{\delta}_{i j}^{q} \equiv\left\{\left(\delta_{i j}^{q}\right)_{L L}\left(\delta_{i j}^{q}\right)_{R R}\right\}^{1 / 2}
\end{aligned}
$$

where $q=u, d$. Note that the absence of the superscript 0 above $\tilde{M}$ indicates that the scalar mass matrices are to be evaluated in the quark mass eigenstate basis. The $\delta$ parameters corresponding to 1-2 and 1-3 scalar mass matrix elements are constrained by neutral pseudoscalar meson mixing to be less than $10^{-1}-10^{-3}$, depending on the superparticle mass spectrum. Typical upper bounds are given in Table 3 . 


\begin{tabular}{l|lll}
\hline \hline & $\left(\delta_{12}^{d}\right)_{L L}$ & $\left(\delta_{13}^{d}\right)_{L L}$ & $\left(\delta_{12}^{u}\right)_{L L}$ \\
\hline Exp. upper bound & $4.0 \times 10^{-2}$ & $9.8 \times 10^{-2}$ & $1.0 \times 10^{-1}$ \\
\hline Prediction of the model & $\lambda^{5}$ & $\lambda^{3}$ & $\lambda^{6}$ \\
& $\sim 5.2 \times 10^{-4}$ & $\sim 1.1 \times 10^{-2}$ & $\sim 1.1 \times 10^{-4}$ \\
\hline \hline & $\left(\delta_{12}^{d}\right)_{R R}$ & $\left(\delta_{13}^{d}\right)_{R R}$ & $\left(\delta_{12}^{u}\right)_{R R}$ \\
\hline Exp. upper bound & $4.0 \times 10^{-2}$ & $9.8 \times 10^{-2}$ & $1.0 \times 10^{-1}$ \\
\hline Prediction of the model & $(\zeta-1) \lambda^{2}$ & $(\zeta-1) \lambda^{3 / 2}$ & $\lambda^{6}$ \\
& $\sim 1.5 \times 10^{-2}$ & $\sim 3.1 \times 10^{-2}$ & $\sim 1.1 \times 10^{-4}$ \\
\hline \hline & $\bar{\delta}_{12}^{d}$ & $\bar{\delta}_{13}^{d}$ & $\bar{\delta}_{12}^{u}$ \\
\hline Exp. upper bound & $2.8 \times 10^{-3}$ & $1.8 \times 10^{-2}$ & $1.7 \times 10^{-2}$ \\
\hline Prediction of the model & $\sqrt{\zeta-1} \lambda^{7 / 2}$ & $\sqrt{\zeta-1} \lambda^{9 / 4}$ & $\lambda^{6}$ \\
& $\sim 2.7 \times 10^{-3}$ & $\sim 1.8 \times 10^{-2}$ & $\sim 1.1 \times 10^{-4}$ \\
\hline \hline
\end{tabular}

Table 3: Upper bounds on $\left(\delta_{i j}^{q}\right)_{L L, R R}$ and $\bar{\delta}_{i j}^{q}$ [10]. Here, we take all the squark and gluino masses to be $500 \mathrm{GeV}$. For comparison, we also show the prediction of our model with $\lambda=0.22$ and $\zeta=c_{3}^{\prime \prime} / c_{0}^{\prime \prime}=1.3$.

We see that the off-diagonal elements are small enough to satisfy the experimental constraints, with the parameter $\zeta \equiv c_{3}^{\prime \prime} / c_{0}^{\prime \prime}=1.3$. This ratio is not constrained by the flavor symmetry, and must be mildly adjusted (at the $30 \%$ level) because of the large right-handed 2-3 mixing angle in the down quark Yukawa matrix. This tuning is so mild, we will not let it concern us further. However, one should keep in mind that $\zeta$ may be naturally close to one if the model is embedded into a larger non-Abelian flavor group at a high scale. Finally, we note that the constraint on 2-3 mixing from $b \rightarrow s \gamma$ is very weak; $\left(\delta_{23}^{d}\right)_{L L, R R} \sim \mathcal{O}(1)$ is allowed [10]. We conclude that the non-Abelian model presented in this section is consistent with the flavor changing neutral current constraints. Note that the model can be extended trivially to the lepton sector by choosing the lepton transformation properties to be identical to those of the down quarks. Then the differences between the down quark and lepton masses can be explained by fluctuations in the order one coefficients. 


\section{Abelian Model}

We have seen that it is possible to construct models with non-Abelian flavor group factors in which flavor symmetries are broken via the dynamics of confinement. Non-Abelian theories greatly alleviate the supersymmetric flavor-changing problem by imposing a natural degeneracy between the first two generation scalar masses in the flavor symmetric limit. In this section, we show that models based on Abelian flavor symmetries can also incorporate our mechanism. Such models solve the supersymmetric flavor problem by arranging an alignment of the quark and squark mass matrices, so that the squark masses are nearly diagonal in the quark mass eigenstate basis. The alignment is strongest in the down quark sector, where the phenomenological constraints are most powerful, and the Cabibbo angle originates in the up quark sector. In this section, we will not present an exhaustive phenomenological analysis, but simply show that our symmetrybreaking mechanism can be combined with the prototypical alignment models of Nir and Seiberg [11.

The important feature of the models of Ref. [11], as well as similar models in Ref. [12], is the presence of two flavon fields, that transform under two independent U(1) flavor symmetries: $S_{1}(-1,0)$ and $S_{2}(0,-1)$. These fields are assumed to acquire vevs

$$
\left\langle S_{1}\right\rangle=\epsilon_{1} \sim \lambda^{2} \quad \text { and } \quad\left\langle S_{2}\right\rangle=\epsilon_{2} \sim \lambda^{3}
$$

Two models using these flavons are presented in Ref. [11 (models A and B) which differ only in the flavor quantum number assignments of the matter fields. We will explicitly consider model A below.

The most elegant way of embedding this flavor sector into the $\mathrm{SU}(3)$ theory described in the previous section is to choose $S_{1}$ to be one of the meson fields, and $S_{2}$ to be one of the baryons. Since the baryon $S_{2}$ has one additional preon, its symmetry breaking effect will be suppressed relative to the meson $S_{1}$ by one factor of $\Lambda / M_{*}$. If we again choose this ratio to be the Cabibbo angle $\lambda$, we account for Eq. (4.1) in a natural way. The two U(1) factors can be taken such that

$$
\begin{aligned}
& Q_{I}=\sqrt{3} Q_{\bar{p}} \\
& Q_{I I}=-Q_{B}
\end{aligned}
$$


where the charges $Q_{\bar{p}}$ and $Q_{B}$ are defined as in the previous section. We can then make the identification

$$
\begin{gathered}
S_{1}(-1,0) \equiv \sigma \\
S_{2}(0,-1) \equiv B
\end{gathered}
$$

The remaining composites $S, A, \phi, \tilde{\phi}$ and $\bar{B}$, also have flavor quantum numbers, and may alter the Yukawa matrices slightly from the form presented in Ref. [11. However, we will now show that the quark-squark alignment remains unaffected. We will assume that the flavor $\mathrm{SU}(2)$ of the previous section is a good flavor symmetry (even though the matter fields are $\mathrm{SU}(2)$ singlets). Since the matter fields will have integral charges under the two $U(1)$ factors, the lowest order combinations of the remaining composites that can contribute to the Yukawa textures are:

$$
\bar{B}(0,+1) \sim \epsilon_{2} \quad \text { and } \quad A^{2}(+1,0) \sim \epsilon_{1}^{2}
$$

Note that we have neglected terms involving $S$ which does not acquire a vev at lowest order. The combination $(\phi \tilde{\phi})^{2} \sim(-1,0)$ couples in the same way as $S_{1}$, but is of higher order in $1 / M_{*}$ and can also be neglected. In model $\mathrm{A}$, the matter fields are assigned charges

$$
\begin{array}{ccc}
Q_{1}(3,-1,+) & Q_{2}(1,0,-) & Q_{3}(0,0,+) \\
U_{1}(-3,3,+) & U_{2}(-1,1,+) & U_{3}(0,0,+) \\
D_{1}(-3,3,+) & D_{2}(1,0,-) & D_{3}(1,0,-)
\end{array}
$$

where the third entry is the charge under our anomalous $Z_{2}$ factor, defined in the previous section. The original textures for Model A in Ref. [11]

$$
h_{u} \sim\left(\begin{array}{ccc}
\epsilon_{2}^{2} & \epsilon_{1}^{2} & 0 \\
0 & \epsilon_{2} & \epsilon_{1} \\
0 & 0 & 1
\end{array}\right) \quad h_{d} \sim\left(\begin{array}{ccc}
\epsilon_{2}^{2} & 0 & 0 \\
0 & \epsilon_{1}^{2} & \epsilon_{1}^{2} \\
0 & \epsilon_{1} & \epsilon_{1}
\end{array}\right)
$$

become

$$
h_{u} \sim\left(\begin{array}{ccc}
\epsilon_{2}^{2} & \epsilon_{1}^{2} & \epsilon_{1}^{6} \epsilon_{2} \\
\epsilon_{1}^{4} \epsilon_{2}^{3} & \epsilon_{2} & \epsilon_{1} \\
\epsilon_{1}^{9} \epsilon_{2}^{3} & \epsilon_{1}^{5} \epsilon_{2} & 1
\end{array}\right) \quad h_{d} \sim\left(\begin{array}{ccc}
\epsilon_{2}^{2} & \epsilon_{1}^{7} \epsilon_{2} & \epsilon_{1}^{7} \epsilon_{2} \\
\epsilon_{1}^{4} \epsilon_{2}^{3} & \epsilon_{1}^{2} & \epsilon_{1}^{2} \\
\epsilon_{1}^{9} \epsilon_{2}^{3} & \epsilon_{1} & \epsilon_{1}
\end{array}\right)
$$


The scalar mass matrices are not holomorphic functions of the flavon fields, so their textures remain unchanged:

$$
\begin{aligned}
& \frac{\left(\tilde{M}_{q}^{2}\right)_{L L}^{0}}{\tilde{m}^{2}} \sim\left(\begin{array}{ccc}
1 & \epsilon_{1}^{2} \epsilon_{2} & \epsilon_{1}^{3} \epsilon_{2} \\
\epsilon_{1}^{2} \epsilon_{2} & 1 & \epsilon_{1} \\
\epsilon_{1}^{3} \epsilon_{2} & \epsilon_{1} & 1
\end{array}\right) \quad \frac{\left(\tilde{M}_{u}^{2}\right)_{R R}^{0}}{\tilde{m}^{2}} \sim\left(\begin{array}{ccc}
1 & \epsilon_{1}^{2} \epsilon_{2}^{2} & \epsilon_{1}^{3} \epsilon_{2}^{3} \\
\epsilon_{1}^{2} \epsilon_{2}^{2} & 1 & \epsilon_{1} \epsilon_{2} \\
\epsilon_{1}^{3} \epsilon_{2}^{3} & \epsilon_{1} \epsilon_{2} & 1
\end{array}\right) \\
& \frac{\left(\tilde{M}_{d}^{2}\right)_{R R}^{0}}{\tilde{m}^{2}} \sim\left(\begin{array}{ccc}
1 & \epsilon_{1}^{4} \epsilon_{2}^{3} & \epsilon_{1}^{4} \epsilon_{2}^{3} \\
\epsilon_{1}^{4} \epsilon_{2}^{3} & 1 & 1 \\
\epsilon_{1}^{4} \epsilon_{2}^{3} & 1 & 1
\end{array}\right)
\end{aligned}
$$

If we now go to the quark mass eigenstate basis, all the rotations on the lefthanded quark fields that are induced by the additional entries in Eq. (4.7) do not alter the order of magnitude of any off-diagonal squark mass matrix elements. Only the 1-2 rotation in the right-handed down sector is large enough to change the $(1,2)$ entry of $\left(\tilde{M}_{d}^{2}\right)_{R R}$ from $\epsilon_{1}^{4} \epsilon_{2}^{3}$ to $\epsilon_{1}^{2} \epsilon_{2}^{3} \sim 10^{-9}$. The bound on this entry from flavor changing neutral currents is of order $10^{-2}$, and is still easily satisfied in the modified model.

Thus, the presence of additional flavons implied by our symmetry-breaking mechanism does not disturb the quark-squark alignment. One can easily verify that the same is true for Model B of Ref. [11] as well.

\section{Conclusions}

Supersymmetric theories have two sets of small dimensionless flavor parameters: one describes the quark and lepton mass ratios and mixing angles, while the other describes squark and slepton non-degeneracies and mixings, which are constrained from flavor-changing processes. We have described a general framework of theories with a flavor symmetry, and given two explicit realistic models, where

- Flavor symmetry breaking is forced by strong supersymmetric gauge interactions.

- All non-zero vevs have a magnitude of order the $\Lambda$ parameter of the new strong gauge force. All flavor symmetry breaking occurs at a single scale, and there is a single small dimensionless parameter, $\Lambda / M_{*}$, where $M_{*}$ is the cutoff for the theory. 
- The flavor symmetry allows certain higher dimension F and D operators coupling quarks $(q)$, Higgs $(H)$ and preons $(p)$,

$$
\left[\bar{q} q H\left(p / M_{*}\right)^{n}\right]_{F} \quad \text { and } \quad\left[q^{\dagger} q\left(p / M_{*}\right)^{m}\right]_{D}
$$

generating small entries in the quark and squark mass matrices of order $\left(\Lambda / M_{*}\right)^{n}$ and $\left(\Lambda / M_{*}\right)^{m}$ respectively.

- While flavor symmetry breaking is forced, there is a large vacuum degeneracy - the quark and squark mass matrices are functions on moduli space. This degeneracy can be lifted in a favorable direction by the combined use

of the $X$ fields and soft, positive supersymmetry breaking squared masses.

\section{Acknowledgments}

We would like to thank N. Arkani-Hamed, H. Murayama and J. Terning for useful discussions. This work was supported in part by the Director, Office of Energy Research, Office of High Energy and Nuclear Physics, Division of High Energy Physics of the U.S. Department of Energy under Contract DE-AC03-76SF00098. LJH was also supported in part by the National Science Foundation under grant PHY-95-14797.

\section{References}

[1] See, for example, H. Georgi, Weak Interactions and Modern Particle Theory, Benjamin/Cummings, Menlo Park, (1984), and references therein.

[2] P.W. Higgs, Phys. Lett. 12, 132 (1964); Phys. Rev. Lett. 13, 508 (1964); Phys. Rev. 145, 1156 (1966); F. Englert and R. Brout, Phys. Rev. Lett. 13, 321 (1964); T.W.B. Kibble, Phys. Rev. 155, 1554 (1967).

[3] L. Ibanez, G.G. Ross, Phys .Lett. B110, 215 (1982); K. Inoue, A. Kakuto, H. Komatsu, and S. Takeshita, Prog. Theor. Phys. 68927 (1982).

[4] N. Seiberg, Phys. Rev. D49, 6857 (1994).

[5] H.-C. Cheng, FERMILAB-PUB-97-019-T, Jan 1997, hep-ph/9702214.

[6] I. Affleck, M. Dine, and N. Seiberg, Nucl. Phys. B256, 557 (1985). 
[7] O. Aharony, J. Sonnenschein, M.E. Peskin, S. Yankielowicz, Phys. Rev. D52, 6157 (1995).

[8] M. Dine, R. Leigh, and A. Kagan, Phys .Rev. D48, 4269 (1993).

[9] R. Gupta and T. Bhattacharya, Phys. Rev. D55, 7203 (1997).

[10] See, for example, F. Gabbiani, E. Gabrielli, A. Masiero, and L. Silvestrini, Nucl. Phys. B477, 321 (1996).

[11] Y. Nir and N. Seiberg, Phys. Lett. B309, 337 (1993)

[12] M. Leurer, Y. Nir, and N. Seiberg, Nucl. Phys. B398, 319 (1993); M. Leurer and N. Seiberg, Nucl. Phys. B420, 468 (1994). 